\title{
Proctocolectomía restauradora. A propósito de 2 casos en un hospital general.
}

Restorative Proctocolectomy. About 2 cases at a general hospital.

Borda Mederos Luis Augusto', Manzaneda Pineda Ana Josefina², Pinto Elera Jesús Omar Andrés².

\section{RESUMEN}

Se presentan 2 pacientes sometidos a proctocolectomía restauradora, el primero con colitis ulcerosa y el segundo con poliposis adenomatosa familiar. Se discuten la efectividad de la técnica, el seguimiento a corto plazo de estos pacientes y las indicaciones para proctocolectomía restauradora.(Rev Med Hered 2009;20:230-234).

PALABRAS CLAVE: Proctocolectomía restauradora, colitis ulcerosa, poliposis adenomatosa de colon, reservorio ileal.

\section{SUMMARY}

There appear 2 patients undergoing restorative proctocolectomyn, the first one with Ulcerative Colitis and the second one with Familial Adenomatous Polyposis. The efficiency of the skill, the short-term follow-up of these patients and the indications for Restorative Proctocolectomy are discussed. (Rev Med Hered 2009;20:230-234).

KEYWORDS: Restorative proctocolectomy, ulcerative colitis, adenomatous polyposis coli, ileal pouch.

1 Cirujano General y Colorectal del Departamento de Cirugía General del Hospital Nacional Guillermo Almenara Irigoyen. EsSalud. Lima, Perú.

2 Alumno de la Facultad de Medicina Alberto Hurtado, Universidad Peruana Cayetano Heredia. Lima, Perú. 


\section{INTRODUCCIÓN}

La Proctocolectomía Restauradora (PR) es un paso evolutivo en la tentativa del cirujano de remover la mucosa colorectal precancerosa o seriamente enferma mientras el paciente mantiene lo mayor posible la continencia anal $(1,2,3)$. Consiste en la colectomía total, protectomía proximal completa, protectomía mucosa distal y anastomosis íleoanal con reservorio ileal $(1,2)$. En diversas instituciones se han realizado modificaciones técnicas al procedimiento, pero los componentes individuales siguen siendo esencialmente iguales a los descritos originalmente por Parks y Nicholls, en 1978.

Las principales indicaciones actuales para realizar PR son colitis ulcerosa (CU) y poliposis adenomatosa familiar (PAF), enfermedades poco frecuentes en Perú.

En nuestro país no existen reportes sobre PR. Nuestro objetivo fue presentar los resultados de la proctocolectomía restauradora realizados en dos pacientes en el Hospital Nacional Guillermo Almenara Irigoyen (HNGAI)-EsSalud, Lima, Perú en el año 2007.

\section{Caso clinico 1}

Paciente de sexo masculino, 56 años de edad, natural de Lima, procedente de Lima, con diagnóstico endoscópico e histopatológico de CU. Presentó durante el año previo a la cirugía, episodios de diarrea mucosanguineolenta (10-20 cámaras diarias), dolor abdominal, tenesmo y pérdida de peso $(20 \mathrm{~kg}$ en un año); los síntomas comenzaron de forma insidiosa y siguieron un curso intermitente, con exacerbaciones y remisiones parciales relacionadas al tratamiento médico recibido en el Hospital Central FAP y posteriormente en el Servicio de Gastroenterología del HNGAI, debido a la falta de respuesta adecuada al tratamiento médico con sulfasalazina, corticoides orales, corticoides endovenosos y azatioprina a dosis plenas fue transferido al Servicio de cirugia para tratamiento quirúrgico.

El paciente fue programado para cirugía electiva.Un día antes de la cirugia se realizó preparación mecánica de colon (polietilenglicol).La PR se ejecutó según la técnica descrita originalmente $(1,2)$,se utilizó profilaxis antibiótica y antitrombótica.

El tiempo operatorio fue de 270 minutos, sangrado operatorio 300 cc, no requirió transfusión sanguínea. Durante el acto operatorio no hubo complicaciones.
La estancia postoperatoria fue de 10 días, durante la cual se administraron analgésicos (tramadol y paracetamol) y antibióticos por 7 días (metronidazol y ciprofloxacino, vía endovenosa). No requirió soporte nutricional especial, inició tolerancia oral al cuarto día y alimentación completa al octavo día. Cierre de ileostomía sin laparotomía a los 3 meses.

El informe de patología fue: Múltiples lesiones erosivas y ulcerativas, algunas sangrantes, se inician en recto y abarcan irregularmente todo el colon. Pólipos hiperplásicos aislados, no se aprecian focos de displasia. Diagnóstico histológico: CU.

Luego del alta el seguimiento se realizó por consultorio externo. El paciente evolucionó favorablemente, recuperó su peso $(60 \mathrm{~kg})$. Al momento de la restitución intestinal (cierre de ileostomía) presentó diarrea acuosa, sin moco, ni sangre (10 cámaras diarias). Ante la posibilidad de pouchitis se administró metronidazol, con poca mejoría por lo que se realizó endoscopia baja y radiografía contrastada del reservorio, descartando la pouchitis. La diarrea mejoró con tratamiento dietético y loperamida.

Durante el primer año de seguimiento presentó 4 ó 5 evacuaciones diarias y su peso se mantuvo adecuado. No manifestó incontinencia anal o disfunción sexual. No hubo necesidad de reintervención.

\section{Caso clínico 2}

Paciente de sexo masculino, 39 años de edad, natural de Pucallpa, procedente de Lima, con diagnóstico de PAF. Seis meses antes de la cirugía, presentó rectorragia, que inició de forma brusca y siguió un curso progresivo. Se realizó colonoscopía, encontrándose más de 100 pólipos, y en el estudio genético resultó ser portador del gen APC; confirmando el diagnóstico inicial. Se escogió manejo quirúrgico mediante PR.

Un día antes de la cirugia se realizó preparación mecánica de colon (polietilenglicol).La PR se ejecutó según la técnica descrita originalmente $(1,2)$,se utilizó profilaxis antibiótica y antitrombótica.

El tiempo operatorio fue de 200 minutos, sangrado operatorio de 400 cc, no requirió transfusión sanguínea. Durante el acto operatorio no hubo 
complicaciones. La estancia postoperatoria fue de 12 días, durante la cual se administraron analgésicos (tramadol y paracetamol) y antibióticos (metronidazol y ciprofloxacino, vía endovenosa). Inició tolerancia oral al quinto día, y al sétimo día alimentación completa. Cierre de ileostomía sin laparotomía a los 3 meses.

El informe de patología: Múltiples pólipos adenomatosos en todo el colon y recto, más de 100. Diagnóstico histológico: PAF.

Luego del alta, el seguimiento se realizó por consultorio externo, primero mensualmente y luego trimestralmente, con tomografía computarizada abdominopélvica, antígeno carcinoembrionario y colonoscopía. La evolución fue favorable, no hubo recurrencia de la enfermedad, evidencia de cáncer colorectal, incontinencia anal o disfunción sexual. No hubo necesidad de reintervención.

\section{DISCUSIÓN}

La colitis ulcerosa (CU) es la principal indicación de PR. Es una enfermedad inflamatoria de la mucosa colorrectal, comienza a nivel del recto y progresa en forma variable hacia el colon. El riesgo de cáncer colorectal en pacientes con CU es mayor; a los 10 años de enfermedad es de 5-10\% y a los 30 años llega al $20 \%$ (4). La cirugía de urgencia está indicada cuando existe hemorragia masiva, megacolon tóxico, perforación o colitis severa que no responde a terapia médica (5). La cirugía electiva está indicada para pacientes con cáncer o displasia, falla o complicaciones del tratamiento médico, y para corregir malnutrición o retardo del crecimiento en niños (5). Nuestro paciente fue sometido a cirugía electiva por presentar CU refractaria a tratamiento médico con sulfasalazina, corticoides orales, corticoides endovenosos y azatioprina. Si bien se encuentra indicado el uso de infliximab en el manejo de CU refractaria a tratamiento médico (5), no se utilizó porque en el año 2007 no estaba disponible en el petitorio farmacológico de EsSalud y el paciente no podía adquirirlo de manera particular debido al alto costo.

Poliposis adenomatosa familiar (PAF) es un síndrome hereditario autosómico dominante, con cerca de $100 \%$ de penetrancia, causado por la mutación del gen adenomatous polyposis coli (APC). Se caracteriza por el desarrollo de múltiples pólipos premalignos en el colon, que en casi el 100\% de los pacientes evoluciona a cáncer colorectal (6). En PAF se indica cirugía una vez hecho el diagnóstico o cuando el paciente es portador del gen APC y empieza a desarrollar pólipos (7); nuestro paciente fue sometido a cirugía luego de realizar colonoscopía con evidencia de más de 100 pólipos y examen genético con diagnóstico final de PAF. PR es también el procedimiento quirúrgico de elección para el tratamiento de pacientes con PAF. En éstos pacientes, el carcinoma inferior de recto y los tumores desmoides intraabdominales son contraindicaciones para PR (8).

Para el manejo quirúrgico de nuestros pacientes con CU y PAF se eligió PR por ser un procedimiento curativo que evita la ileostomía permanente, preserva la función anorectal y elimina el riesgo de cáncer colorectal $(9,10)$. Existen reportes de otros procedimientos para el manejo de estas enfermedades como la colectomía con ileostomía continente permanente, cuyo dispositivo de ileostomía debe ser vaciado entre 4-8 veces y en algunos casos se requiere revisión quirúrgica del estoma, existen complicaciones de la herida pélvica, se presenta obstrucción intestinal, disfunción vesical o disfunción sexual (1); por otro lado la colectomía total con anastomosis ileorectal conserva la función del esfínter anal y no presenta las complicaciones del anterior procedimiento, sin embargo, no es curativa y requiere seguimiento por riesgo de cáncer o de enfermedad inflamatoria en el remanente rectal (1).

El procedimiento quirúrgico fue en 2 tiempos, el tiempo abdominal y el perineal; en este último se realizó mucosectomía anorectal distal, para disminuir el riesgo de displasia y carcinoma en curso en la mucosa rectal, pese al posible daño del esfínter anal (11). Construimos el reservorio ileal en "J" porque es fácil de realizar con dispositivos convencionales de autosutura o manualmente, se adapta bien a la pelvis menor, se vacía fácilmente y presenta resultados funcionales similares a las otras configuraciones "S" y "W" (7). El nuevo recto, constituido por el reservorio ileal, se anastomosó al ano manualmente; escogimos esta técnica para prevenir el riesgo de displasia a largo plazo asociado al uso de la engrapadora, ya que ambas técnicas presentan resultados similares (12). Se realizó ileostomía protectora para evitar el riesgo de sepsis en caso de fugas anastomóticas del reservorio $(7,13)$.

PR es un procedimiento complejo con una tasa de complicaciones de $25-40 \%$, y una tasa de reintervenciones del 15-25\% (14). Así se realice correctamente, la función intestinal no será completamente normal, no obstante, según Pezim y 
Nicholls, la mayoría de pacientes prefieren someterse a PR, por los beneficios sociales, laborales, de imagen corporal y calidad de vida que obtienen al no utilizar un dispositivo de estoma y preservar la función intestinal (1). Las principales complicaciones incluyen hemorragias, obstrucción intestinal, sepsis pélvica, diarrea, esfuerzo para defecar, dolor anal y abdominal, fístulas vaginales, displasia en el reservorio o falla del mismo y pouchitis, la más frecuente a largo plazo $(15,16)$. La diarrea se reporta en $2-9 \%$ de los pacientes operados, el $62 \%$ se presenta luego de la restitución del tránsito intestinal (17). Durante el seguimiento a corto plazo nuestro paciente con CU presentó diarrea (hasta 10 veces al día) luego de la restitución del transito intestinal, se manejaron con tratamiento dietético y loperamida, como está descrito (17).

En la enfermedad de Crohn actualmente se recomienda cirugía segmentaria (7). Antes se realizaba también PR, pero se observaba recurrencia de la enfermedad en el reservorio y complicaciones severas, por lo cual el procedimiento quedó contraindicado (1). En pacientes con colitis indeterminada se puede realizar PR y los resultados son similares a los encontrados en $\mathrm{CU}$, pero con mayor riesgo de complicaciones postoperatorias del reservorio (18). En la enfermedad funcional intestinal (enfermedad de Hirchsprung o megacolon idiopático) se indica PR cuando la anastomosis ileorectal es insuficiente debido a un compromiso total del colon o cuando se requiere una ileostomía permanente (10).

Existe un reporte de PR en 13 pacientes con estreñimiento, pese a la alta tasa de complicaciones, el 85\% de pacientes mostraron una mejoría en sus síntomas y su calidad de vida (19). Los pacientes con mala función del esfínter anal están contraindicados a PR (20). La edad no es un impedimento para realizar PR, sin embargo, los pacientes mayores de 50 años comienzan a presentar problemas de continencia por lo cual algunos cirujanos solo recomiendan PR hasta esa edad (20).

La PR es un procedimiento efectivo para el tratamiento quirúrgico de pacientes con $\mathrm{CU}$ y PAF; cura la enfermedad, previene la aparición de cáncer colorectal y mantiene la calidad de vida de los pacientes.

\section{Correspondencia:}

Ana Josefina Manzaneda Pineda

Dirección: Talara 402, Jesús María

Teléfono: 511-993601354

Correo electrónico: ana.manzaneda@upch.pe

\section{REFERENCIAS BIBLIOGRÁFICAS}

1. Nicholls J, Bartolo D, Mortensen N.(Edit). Restorative Proctocolectomy. Oxford: Blackwell Scientific Publications 1993. p. 1-17.

2. Nicholls RJ. Restorative proctocolectomy with various types of reservoir. World J Surg 1987;11:751-762.

3. Nicholls RJ, Kamm MA. Proctocolectomy wih restorative ileo-anal reservoir for severe idiopathic constipation. Report of two cases. Dis Colon Rectum 1988;31(12):968-969.

4. Khorrami Mashhadi S, Trapero M, Gisbert J P, Gómez Domínguez E, Maté-Jiménez J. A pilot study on the endoscopic surveillance of colorectal dysplasia and cancer in long-standing ulcerative colitis. Rev esp enferm dig 2005;97:16-23.

5. Wolff B, Fleshman, Beck d, Pemberton J, Wexner S. The ASCRS Text book of Colon Rectal Surgery. 1ra edición. New York: Springer; 2007. p. 559-563.

6. Cosme A, Castiella A. Poliposis gastrointestinales hereditarias. En: Actualizaciones temáticas en gastroenterología. Madaus S.A.; 1999. p. 1-60.

7. Brunicardi FC. Schawrtz Principios de Cirugía. 8ava edición. México D.F.: McGraw-Hill Interamericana; 2006. p. 1072, 1080, 1081, 1085.

8. Nandakumar G, Morgan JA, Silverberg D, Steinhagen RM. Familial polyposis coli: clinical manifestations, evaluation, management and treatment. Mt Sinai J Med 2004;71(6):384-391.

9. Kelly KA, Dozois RR. Chronic ulcerative colitis. En: Kelly KA, Sarr MG, Hinder RA, eds. Mayo Clinic Gastrointestinal Surgery. Philadelphia:WB Saunders; 2004. p. 533-552.

10. Dozois EJ, Dozois RR. Familial adenomatous polyposis. En: Kelly KA, Sarr MG, Hinder RA, eds. Mayo Clinic Gastrointestinal Surgery. Philadelphia: WB Saunders; 2004. p. 559-567.

11. Regimbeau J, Panis Y, Pocard M, Hautefeuille P, Valleur P. Handsewn ileal pouch-anal anastomosis on the dentate line after total proctectomy: Technique to avoid incomplete mucosectomy and need for long-term follow up of the anal transition zone. Dis colon Rectum 2001;44(1):43-50.

12. Lovegrove R, Constantinides V, Heriot A, Athanasiou T, Darzi A, Remzi F, et al. A comparison of hand-sewn versus stapled ileal pouch anal anastomosis (IPAA) following proctocolectomy: a meta-analysis of 4183 patients. Ann Surg 2006;244(1):18-26. 
13. Weston-Petrides G, Lovegrove R, Tilney H, Heriot A, Nicholls J, Mortensen N, et al. Comparison of outcomes after restorative proctocolectomy with or without defunctioning ileostomy. Arch Surg 2008;143(4):406412.

14. Dozois RR, Goldberg SM, Rothenburger DA, et al. Restorative proctocolectomy wih ileal reservoir. Int J Colorectal Dis 1986;1:2-19.

15. Bach S, Mortensen N. Revolution and Evolution: 30 years of ileoanal pouch surgery. Inflamm Bowel Dis 2006;12(2):131-145.

16. Yu ED, Shao Z, Shen B. Pouchitis. World J Gastroenterol 2007;13(42): 5598-5604.
17. Galandiuk S, Scott N, Dozois R, et al. Ileal pouch-anal anastomosis. Reoperation for pouch-related complications. Ann Surg 1990;212(4):446-454.

18. Mitchell PJ, Rabau MY, Haboubi NY. Indeterminate colitis. Tech Coloproctol 2007;11(2):91-96.

19. Hosie KB, Kmiot WA, Keighley MR. Constipation: Another indication dor restorative proctocolectomy. Br J Surg 1990;77(7):801-802.

20. Senninger N, Brüwer M. Indications for restorative proctocolectomy in systemic colorectal diseases (ulcerative colitis and familial adenomatous polyposis coli). Langenbecks Arch Chir Suppl Kongressbd 1998;115:468-471. 